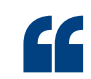

MIEF1 ... has

a dual role in inhibiting fission and promoting fusion.

\title{
MIEF1 mingles with mitochondria
}

The morphology of mitochondria, which is determined by a dynamic cycle of membrane fusion and fission events, is crucial for normal functioning of this organelle. Zhao et al. have now identified a new component of the human mitochondrial outer membrane, mitochondrial elongation factor 1 (MIEF1), which has a dual role in inhibiting fission and promoting fusion.

After identifying MIEF1 as an integral protein at the mitochondrial outer membrane, Zhao et al. observed that overexpression of MIEF1 in $293 \mathrm{~T}$ cells increased mitochondrial

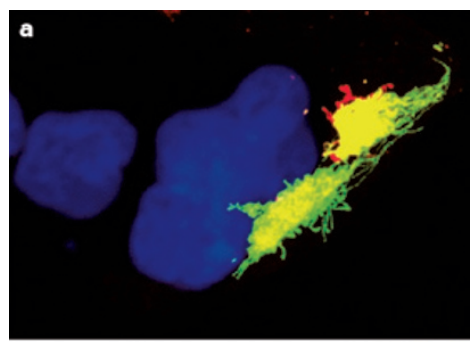

b

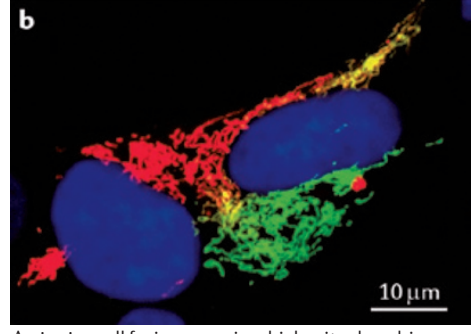

An in vivo cell fusion assay in which mitochondria are labelled in red or green, with mitochondrial fusion indicated in yellow. Polykaryon cells (nuclei labelled in blue) expressing tagged MIEF1 (a) showed increased mitochondrial fusion compared with control cells expressing an empty vector (b). Image is reproduced, with permission, from Zhao, J. et al. @ (2011) Macmillan Publishers Ltd. All rights reserved. fusion, resulting in an elongated morphology. This was also seen when MIEF1 was overexpressed in an in vivo cell fusion assay. Conversely, RNA interference-mediated depletion of MIEF1 increased mitochondrial fragmentation. These effects on mitochondrial morphology were independent of mitofusin 2 (MFN2), a well-characterized regulator of the fusion pathway.

Mitochondrial fission requires the GTPase dynamin-related protein 1 (DRP1; also known as DNM1L), which predominantly localizes to the cytoplasm and must be actively recruited to the mitochondrial membrane. In yeast, mitochondrial fission 1 (Fis1) is thought to act as its mitochondrial receptor. However, this does not hold true in vertebrates, and efforts to work out how vertebrate DRP1 reaches mitochondria have identified few regulators so far. Therefore, the authors sought to determine whether MIEF1 might be the vertebrate receptor for DRP1. They found that MIEF1 and endogenous DRP1 colocalized on mitochondria and they also associated by co-immunoprecipitation. Moreover, overexpression of MIEF1 resulted in increased association of DRP1 with mitochondria. The authors mapped the region of MIEF1 that is important for this association and showed that overexpressed MIEF1 affects mitochondrial morphology only if it retains this DRP1-binding region. The effects of MIEF1 on
DRP1 recruitment did not require FIS1, MFN2 or mitochondrial fission factor (MFF), another recently identified protein that promotes DRP1 mitochondrial localization.

The fact that MIEF1 recruits DRP1 to mitochondria might suggest that it would promote DRP1-mediated fission of mitochondria. However, Zhao et al. find that MIEF1 impairs DRP1 binding to GTP, which would be predicted to inhibit DRP1's effects on fission. Co-immunoprecipitation analysis showed that MIEF1 also associates with FIS1, independently of DRP1 binding, and increased levels of FIS1 partially reverted the effects of MIEF1 overexpression on fusion. Thus, MIEF1 may promote mitochondrial fusion through a mechanism that is independent of MFN2 but that is kept in check by FIS1.

MIEF1 seems to provide a means of regulating mitochondrial dynamics that is unique to vertebrates. It will be intriguing to learn more about how it exerts its dual effects on fission and fusion, and how its efforts in recruiting DRP1 are coordinated with other factors, such as MFF.

Alison Schuldt

ORIGINAL RESEARCH PAPER Zhao, J. et al. Human MIEF1 recruits Drp1 to mitochondrial outer membranes and promotes mitochondrial fusion rather than fission. EMBO J.

24 Jun 2011 (doi:10.1038/emboj.2011.198) FURTHER READING Westermann, B. Mitochondrial fusion and fission in cell life and death. Nature Rev. Mol. Cell Biol. 11, 872-884 (2010) 